\title{
In vitro Epstein-Barr Virus Infection Model of Rabbit Lymphocytes from Peripheral Blood or Spleen
}

\author{
Kyosuke Kanai $^{a, d}$ Kaoru Kato ${ }^{a, c}$ Hitoshi Sano ${ }^{a, b}$ Keiko Nagata ${ }^{a}$ \\ Keisuke Okuno a, b Satoshi Kuwamoto ${ }^{a}$ Hiromi Higaki ${ }^{a}$ Hirotsugu Sugihara ${ }^{a}$ \\ Masako Kato $^{a}$ Ichiro Murakami $^{a}$ Kazuhiko Hayashi ${ }^{a}$ \\ Divisions of a Molecular Pathology and ${ }^{b}$ Pediatrics and Perinatology, Faculty of Medicine, Tottori University, \\ Yonago, 'Kato Animal Hospital, Tottori, and d Department of Virology I, National Institute of Infectious Diseases, \\ Tokyo, Japan
}

\section{Key Words}

Epstein-Barr virus I - In vitro infection model ·

EBV-associated diseases

\begin{abstract}
Most humans become lifelong carriers of Epstein-Barr virus (EBV) by adulthood. Primary EBV infection in adolescents causes infectious mononucleosis. EBV infection is associated with various diseases, neoplasms and hematological disorders. Recently, we reported that EBV can infect rabbits by intravenous, intranasal and/or peroral inoculation, which caused primary EBV infection in rabbits with heterogeneous host reactions. Some rabbits showed chronic and lifelong EBV infection with hemophagocytosis. In this study, to reveal detailed mechanisms in rabbit EBV infection, an in vitro investigation was performed. We elucidated that: (1) EBV can infect rabbit peripheral blood mononuclear cells and splenic lymphocytes in vitro, because EBV gene expressions were confirmed. (2) It is highly likely that the B cell is the main target cell of rabbit EBV infection and is immortalized similar to humans. (3) CD8+ T cells increased in the rabbit in vivo model after EBV inoculation, whereas an increase of B cells occurred after their transient decrease. These data suggest that EBV-infected B cells were proliferated, while CD8+ T cells increased to recognize and kill them. This system may explain
\end{abstract}

the paths of rabbit EBV infection and host reaction, simulating human EBV infection. In vitro studies will be helpful to reveal the pathogenesis of rabbit EBV infection and EBV-associated diseases.

Copyright $\odot 2010$ S. Karger AG, Basel

\section{Introduction}

Epstein-Barr virus (EBV) was discovered by Epstein et al. [1]. It is a gamma-herpesvirus belonging to human herpesvirus 4 and infects most humans by adulthood. Most humans are infected in childhood by saliva transfer, and become lifelong carriers of EBV. The major target cells of EBV are B lymphocytes (B cells). A primary infection of EBV with latent type 3 infection is followed by latent type 1 caused by host immunity $[2,3]$. Infected $B$ cells can be immortalized in vitro [4].

Certain patients with an EBV primary infection develop infectious mononucleosis which is resolved spontaneously [5]. However, EBV is capable of occasionally causing severe disease such as EBV-associated hemophagocytic syndrome [6] and chronic active EBV infection [7]. In addition, EBV is known to be a tumorigenic virus and is associated with various tumors, which include Burkitt's lymphoma, Hodgkin's lymphoma, pyo-

\section{KARGER}

Fax +41613061234 E-Mail karger@karger.ch www.karger.com
(C) 2010 S. Karger AG, Basel

0300-5526/11/0541-0017\$38.00/0

Accessible online at:

www.karger.com/int
Kyosuke Kanai, $\mathrm{PhD}$, and Kazuhiko Hayashi, MD

Division of Molecular Pathology, Faculty of Medicine, Tottori University

86 Nishi-cho, Yonago, Tottori 683-8503 (Japan)

Tel. +81 859386063 , Fax +81 859386060

E-Mail kyosuke@gmail.com or hayashik@med.tottori-u.ac.jp 
thorax-associated lymphoma, opportunistic lymphoma including post-transplant lymphoproliferative disorders, some cases of gastric carcinoma, nasopharyngeal carcinoma and other neoplasms $[2,8-10]$. Therefore, it is important to clarify the mechanism and pathogenesis of these tumors for successful treatment.

Investigation of the pathogenesis of EBV has been described in some animal models. EBV infects not only humans but also certain types of New World monkeys. So far, animal models of the common marmoset, cottontop tamarin, and douroucouli have been described [11-13] Some New World monkeys developed post-transplant lymphoproliferative disorder-like B cell lymphoma, infectious mononucleosis-like disease, or latent infection. A rhesus monkey model using a rhesus lymphocryptovirus similar to EBV has been useful for elucidating primary and persistent infection of EBV [14]. However, these monkeys are precious natural resources. Although EBV does not infect mice and severe combined immunodeficiency (SCID) mice in general, it can infect humanized SCID or mice with immunodeficiency such as NOD/SCID, NOD/Shi-scid/IL-2R $\gamma^{\text {null }}$ (NOG), and Rag $2^{-/-} \gamma \mathrm{c}^{-/-}$, and which were transplanted with human hematopoietic stem cells [15-18]. It has been reported previously that rabbits infected with herpesvirus papio (HVP), a type of lymphocryptovirus in baboons, developed virus-associated lymphoproliferative disorders and hemophagocytic syndrome, and are a possible animal model for studying the EBV-associated hemophagocytic syndrome [19-21]. In our previous reports, we recently revealed that EBV could frequently infect rabbit lymphocytes in vivo by intravenous inoculation, which caused a heterogeneous host reaction in the rabbits [22]; some rabbits were also infected via the intranasal or peroral route [23]. In addition, we confirmed that in some of the rabbits inoculated intravenously, EBV-DNA were detected continuously or intermittently lifelong over 1,000 days. Autopsy of rabbits with lifetime EBV infection revealed the infiltration of lymphocytes expressing EBVencoded small RNA 1 (EBER1) or latent membrane protein 1 (LMP1) in the spleen and lymph nodes. Additionally, mild or moderate hemophagocytosis and some large atypical lymphocyte infiltrations were also observed in the autopsied spleens or lymph nodes [unpubl. data]. These results mean that EBV inoculated into rabbits causes transient viral infection and/or is maintained and survives in the rabbits for life without loss of viral function. An EBV-infected rabbit model may be appropriate to investigate in vivo EBV infection and the mechanisms for maintaining EBV. However, details of the cell or mo- lecular biology of EBV-infected rabbits are still unclear. Therefore, in vitro investigations are needed to reveal the detailed mechanism of EBV infection.

In this study, we investigated whether EBV can infect and immortalize rabbit peripheral blood mononuclear cells (PBMC) or splenic lymphocytes in vitro, and tried to determine the subsets of in vitro EBV-infected lymphocytes. Therefore, each abundance and transition of lymphocyte subsets in the rabbit after in vivo EBV inoculation were also analyzed by flow cytometry.

\section{Materials and Methods}

\section{EBV-Producing Cells and Culture}

B95-8, which is an EBV-producing marmoset lymphoid cell line established by infection with a virus derived from a patient with infectious mononucleosis, was cultured in RPMI1640 medium (Nissui, Tokyo, Japan) with 5\% fetal bovine serum (FBS), glutamine, and antibiotics (penicillin-G and streptomycin). B95-8 cells were incubated at $37^{\circ}$ for 1 week and then incubated at $33^{\circ}$ for 2 weeks; cell growth was accelerated at $37^{\circ}$, while EBV production was increased at $33^{\circ}$. Supernatants obtained from the B95-8 culture were filtrated using $0.45-\mu \mathrm{m}$ filters to eliminate the cell debris and were centrifuged at $16,000 \mathrm{~g}$ for $90 \mathrm{~min}$ to remove supernatants by a Compact High Speed Refrigerated Centrifuge 7780 (Kubota, Tokyo, Japan). After centrifugation, the pellets were diluted with the fresh RPMI 1640 medium and stored at $-80^{\circ}$ until use.

Preparations of Rabbit PBMC and Spleen Lymphocytes, and Exposure to $E B V$

The collected peripheral bloods in the tubes with EDTA-2K were diluted with phosphate-buffered saline (PBS) into three times volumes and spleens were dissected out and suspended in PBS. These suspensions were placed on the mixture of Lymphosepar I and Lymphosepar II (Lymphosepar I and Lymphosepar II = 2:1) (Immuno-Biological Laboratories, Takasaki, Japan), and centrifuged at $490 \mathrm{~g}$ for $30 \mathrm{~min}$. The layers of PBMC and/or lymphocytes were collected in the new tube and washed. Rabbit PBMC and/or spleen lymphocytes were exposed for infection by EBV. For exposure to EBV, washed $1 \times 10^{6}$ cells were suspended in $1 \mathrm{ml}$ of EBV suspension as described previously in a $15-\mathrm{ml}$ tube and incubated for $2 \mathrm{~h}$ at $37^{\circ}$ with tapping. Subsequently, cells were washed in PBS, and cultured in fresh 10\% FBS, RPMI1640 medium in $37^{\circ}$ until the optional days.

Detection of EBV-Related Genes mRNA in PBMC by RT-PCR

EBV-exposed rabbit PBMC were cultured and sampled every 2 days until 10, 20 and 30 days, and EBNA1 and ZEBRA mRNA expression were determined. EBV-exposed spleen lymphocytes were cultured and sampled every 2 days until 10 days, and EBNA1, EBNA2 and ZEBRA mRNA expression was analyzed. The RNA was extracted with the RNeasy Micro Kit (Qiagen, Hilden, Germany) and QIA shredder (Qiagen) in accordance with the manufacturer's protocol. RNA from PBMC were quantified by NanoDrop (Thermo Scientific, Wilmington, Mass., USA) with each RNA sample measuring $500 \mathrm{ng}$. RT-PCR was performed with TaKaRa RNA PCR ${ }^{\mathrm{TM}}$ Kit (AMV) version 3.0 (TaKaRa Bio., 
Otsu, Japan) in accordance with its protocol. The mRNA expression of EA, EBNA1, EBNA2, and ZEBRA was investigated by PCR or nested PCR using the described primers [23]. The mRNA of glyceraldehyde-3-phosphate dehydrogenase (GAPDH) was used as the internal control. The PCR mixture comprised $2 \mu \mathrm{l}$ of $10 \times$ Ex Taq buffer, $1.6 \mu \mathrm{l}$ of dNTP Mixture, $0.1 \mu \mathrm{l}$ of TaKaRa Ex Taq HS, $12.3 \mu \mathrm{l}$ of $\mathrm{H}_{2} \mathrm{O}, 1 \mu \mathrm{l}$ of sense primer, $1 \mu \mathrm{l}$ of antisense primer, and $2 \mu \mathrm{l}$ of the sample. BJAB cells exposed to EBV and incubated for 2 days were used as positive control, and nuclease free $\mathrm{H}_{2} \mathrm{O}$ used for mixture dilution was used as negative control.

\section{Immunofluorescence Staining of Rabbit PBMC or Splenic Lymphocytes}

For discrimination of EBV-infected cells, EAD expression in rabbit PBMC and/or spleen lymphocytes were examined by an immunofluorescence staining method. Rabbit spleen lymphocytes were exposed to EBV and cultured for 4 days. Lymphocytes were washed in PBS, smeared on sides, and fixed (acetone:methanol = 1:2). $1 \mu \mathrm{g} / \mathrm{ml}$ sheep IgG, PBS was used for blocking. Then, 1:600 diluted mouse anti-EAD antibody (Millipore, Billerica, Mass., USA) was used as the 1 st antibody after washing, and $0.1 \%$ diluted fluorescein isothiocyanate (FITC)-conjugated anti-mouse IgG sheep antibody (Cappel, West Chester, Pa., USA) was applied as the second antibody. The slides were incubated at $37^{\circ}$ for 30 min after the addition of each antibody, respectively. Finally, PI or DAPI were added for staining of nuclei and incubated at room temperature for $15 \mathrm{~min}$. The slides were then observed under a fluorescence microscope (Olympus, Tokyo, Japan).

To determine the cell type of expressed EAD, double-immunofluorescence staining was performed. 1:200-diluted mouse anti-rabbit CD3 and CD79a (Spring Valley Laboratories, San Diego, Calif., USA) were also used as first antibody and cell type markers, and incubated. A rabbit polyclonal to mouse IgG-F(ab)' 2 fragment (rhodamine) (Abcam, Cambridge, UK) was used as the second antibody and incubated. After 3 times washing in PBS, antiEAD antibody (Millipore) conjugated with biotin by Biotin Labeling Kit- $\mathrm{NH}_{2}$ (Dojindo Laboratories, Kumamoto, Japan) was used as the first antibody for EAD detection, and rabbit polyclonal to biotin (FITC) (Abcam) was used as the second antibody. After each addition of antibody, the slides were incubated at $37^{\circ}$ for $30 \mathrm{~min}$. Finally, after DAPI staining, the slides were observed as before.

\section{Colony Formation Assay of EBV-Exposed Rabbit PBMC}

Methylcellulose medium culture was performed to reveal the immortalization potential of EBV-exposed rabbit PBMC. PBMC obtained from 2 individual rabbits were exposed to EBV for $2 \mathrm{~h}$ and $3 \times 10^{6} \mathrm{PBMC}$ were suspended in $3 \mathrm{ml}$ of RPMI1640 medium containing glutamine, antibiotics, 30\% FBS, $100 \mu \mathrm{M} 2$-mercaptoethanol, and $1.2 \%$ methylcellulose: each $1 \times 10^{6}$ cells plated on 3 dishes and incubated at $37^{\circ}$ for 3 weeks. EBV-exposed human PBMC were plated on 3 dishes as the positive control, and EBV-unexposed PBMC were also plated to use as the negative control. The colonies that appeared were observed under a stereomicroscope (Leica mzfliii R-LS, Leica Microsystems, Wetzlar, Germany).

\section{Intravenous Inoculation with EBV into the Rabbit}

Rabbits were specific-pathogen-free normal male Japanese White Rabbits (weight, $2-3 \mathrm{~kg}$ ) obtained from Shimizu Laboratory Supplies (Kyoto, Japan). The viral solutions of $9.2 \times 10^{5}$ cop- ies of EBV-DNA were inoculated into the rabbit intravenously. The day of viral inoculation was defined as 'week 0', and blood was collected every week.

The animal experiment was carried out under the control of the committee in accordance with 'The Guidelines for Animal Experimentation in the Faculty of Medicine, Tottori University', 'The Law Concerning the Human Care and Control of Animals' (Law No. 105; October 1, 1973) and 'The Japanese Government Notification on Feeding and Safekeeping of Animals' (Notification No. 6, March 27, 1980).

\section{Flow Cytometry Analysis for Lymphocyte Subsets of PBMC}

\section{from EBV-Inoculated Rabbits}

To determine the abundance of rabbit lymphocyte cell types, $\mathrm{B}$ cells, CD4+ and CD8+ T cells, and flow cytometry analysis were carried out in PBMC from EBV-inoculated rabbits. The following antibodies for B cells, CD4+ T cells or CD8+ T cells were used: goat anti-rabbit IgM ( $\mu$ heavy chain specific) with R-phycoerythrin (R-PE) conjugated with B cells (SouthernBiotech, Birmingham, Ala., USA), mouse anti-rabbit CD4 conjugated with FITC (AbD Serotec, Oxford, UK) and mouse anti-rabbit CD8 conjugated with FITC (AbD Serotec). PBMC were separated by the method described previously, $5 \times 10^{5}$ cells were suspended in $10 \%$ rabbit serum, $0.02 \% \mathrm{NaN}_{3}$, Hanks' BSS and incubated at $4^{\circ}$ for $10 \mathrm{~min}$ for blocking. After centrifugation $(490 \mathrm{~g})$ for $5 \mathrm{~min}$, the supernatants were aspirated. Antibodies were diluted by $2 \%$ rabbit serum, $0.02 \% \mathrm{NaN}_{3}$, Hanks' BSS to a final concentration of 0.5 $\mu \mathrm{g}$ in $100 \mu \mathrm{l}$, and added to cells in the tube, suspended and incubated at $4^{\circ}$ for $30 \mathrm{~min}$. Cells were centrifuged and the supernatants were aspirated and $0.2 \mathrm{ng} / 200 \mu \mathrm{l}$ PI was added, suspended and incubated at room temperature for $15 \mathrm{~min}$. Prepared cells were analyzed using EPICS XL (Beckman Coulter, Calif., USA) and obtained data were analyzed by WinMDI version 2.9. The sums of CD4 and CD8 values were considered as value of total T cell in this experiment. The incremental values of each lymphocyte subset that was calculated by data obtained by flow cytometry were shown by relative values which were considered on the principle that the value of day 0 was 100 (fig. 4a).

\section{Real-Time Quantitative PCR of EBV-DNA}

The PCR primers selected for this assay were specific for the $B A L F 5$ gene, which encodes the viral DNA polymerase [24]. The upstream and downstream primer sequences were $5^{\prime}$-CGGAAGCCCTCTGGACTTC-3' and 5'-CCCTGTTTATCCGATG-3', respectively. A fluorogenic probe (5'-TGTACACGCACGAGAAATGCGCC- $3^{\prime}$ ) with a sequence located between the PCR primer sequences was synthesized by PE Applied Biosystems (Foster City, Calif., USA). PCR was performed using the TaqMan PCR kit (PE Applied Biosystems) [25]. Either 250 ng of DNA from peripheral blood mononuclear cells (PBMC), plasma, or culture supernatant was added to the PCR mixture containing $10 \mathrm{mM}$ Tris $(\mathrm{pH}$ 8.3); $50 \mathrm{mM} \mathrm{KCl} ; 10 \mathrm{mM}$ EDTA; $5 \mathrm{mM} \mathrm{MgCl}_{2} ; 400 \mathrm{mM}$ each of dNTP; $0.2 \mathrm{mM}$ each primer; $0.1 \mathrm{mM}$ fluorogenic probe, and 1.25 U AmpliTaq Gold (PE Applied Biosystems). Following a 10min activation of AmpliTaq Gold at $95^{\circ}, 45-50$ cycles of $15 \mathrm{~s}$ at $95^{\circ}$ and $1 \mathrm{~min}$ at $62^{\circ}$ were carried out by a 7700 Sequence Detector (PE Applied Biosystems). A plasmid containing the BALF5 gene (pGEM-BALF5) was used as the positive control. Each sample was tested in duplicate, and the mean of the two values was considered as the copy number of the samples. Samples were de- 


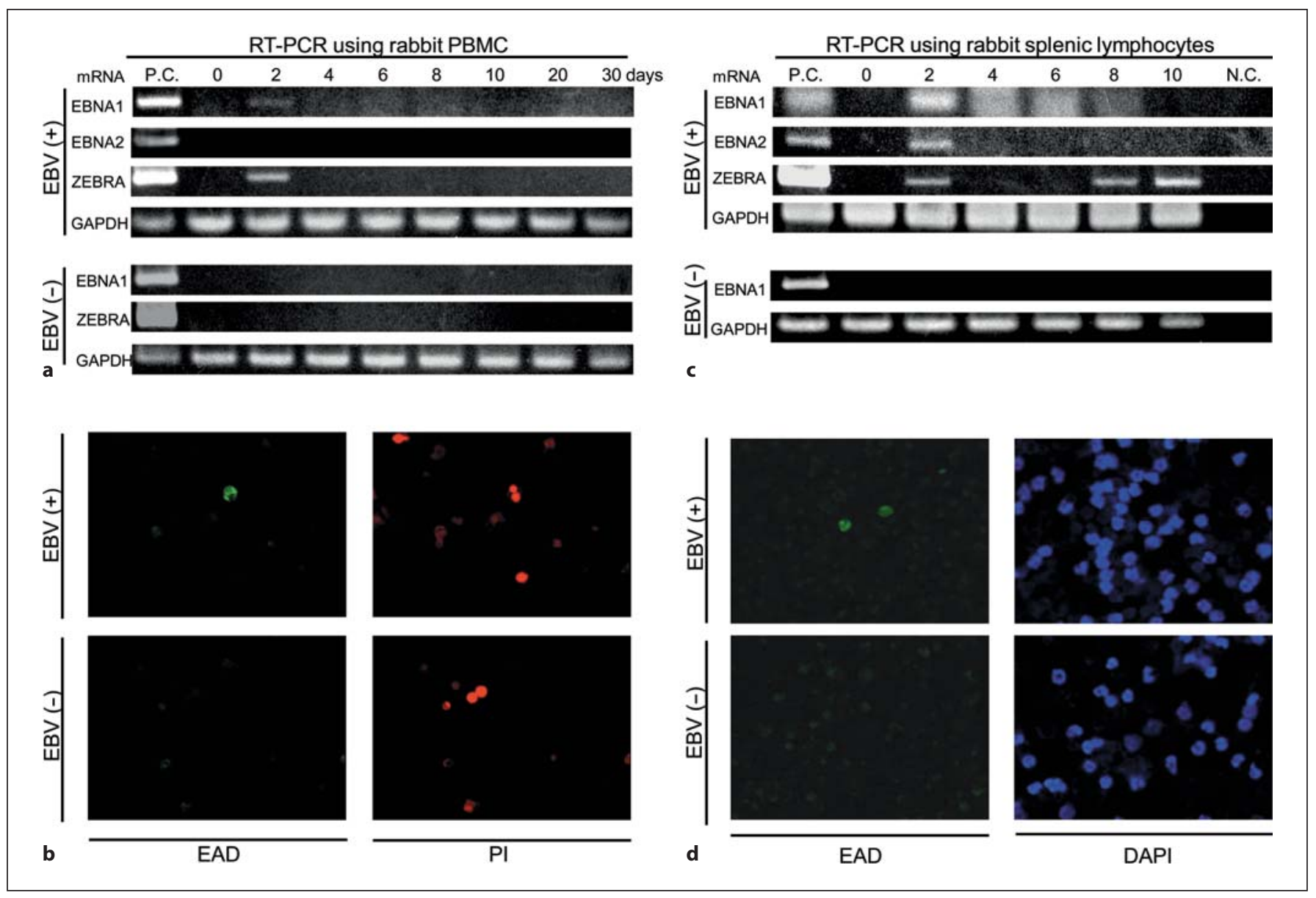

Fig. 1. EBV-encoded genes expression in rabbit PBMC and splenic lymphocytes in vitro. EBV-encoded gene mRNA-specific RTPCR and immunofluorescence staining (IF) of EAD were performed. Both EBNA1- and ZEBRA-mRNA were detected only on day 2 and were not observed later. EBNA2-mRNA were not detected during the observation period (a). No RT-PCR products were detected in the EBV-unexposed PBMC used as negative con-

trol. IF was performed with EBV-inoculated rabbit PBMC in vitro on day 4 after EBV exposure. Very low populations of EAD-expressing cells were observed (b). EBNA1-, EBNA2- and ZEBRAmRNA were expressed on day 2, and disappeared on day 4 . ZEBRA-mRNA reappeared on days 8 and 10 (c). Some EAD-expressing cells were observed by IF in rabbit splenic lymphocytes in vitro on day 4 after EBV exposure (d).

fined as negative if the threshold cycle values exceeded 50. When EBV-DNA copies were lower than the minimal sensitivity $(20$ copies $/ 10^{6} \mathrm{WBCs}$ ), they were considered negative and indicated as $<2 \times 10^{1}$ (fig. 4 ).

\section{Results}

In vitro Investigation of EBV-Encoded Gene

Expression in EBV-Exposed Rabbit PBMC or

Splenic Lymphocytes

EBV-encoded gene mRNA-specific RT-PCR and immunofluorescence staining against EAD, which was the EBV early gene protein of replication phase, were per- formed in EBV-exposed PBMC or splenic lymphocytes in vitro (fig. 1). In PBMC, as a result of RT-PCR, both EBNA1- and ZEBRA-mRNA were detected only on day 2, but not observed on the other days, and EBNA2-mRNA was not detected on the days examined (fig. 1a). No RTPCR products were observed in EBV-unexposed PBMC used as the negative control. The immunofluorescence staining in rabbit PBMC on day 4 after exposure showed a few EAD-expressing cells (fig. 1b). The abundance of EAD-positive cells was lower than $0.2 \%$.

In splenic lymphocytes, RT-PCR revealed expression of EBNA1-, EBNA2- and/or ZEBRA-mRNA on day 2, but not on days 4 and 6 (fig. 1c). Although EBNA1- and EBNA2-mRNA did not appear after day 2, ZEBRA- 


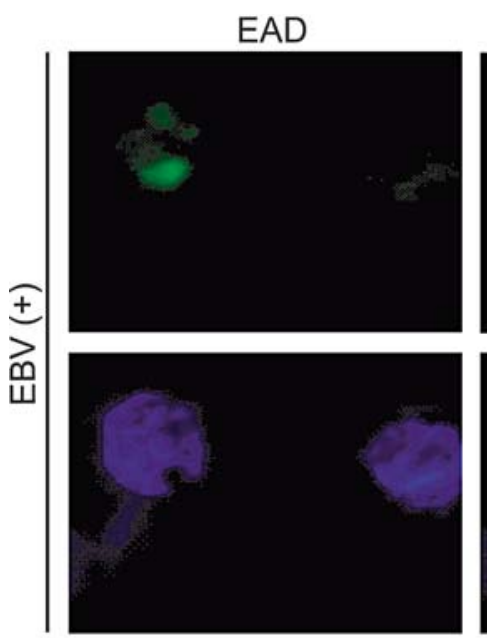

a
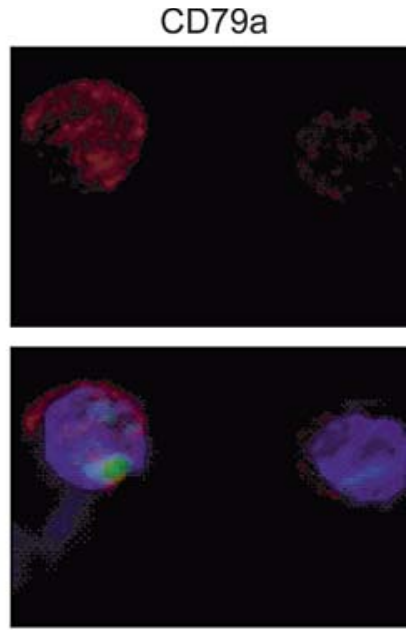

Merge

Fig. 2. Double immunofluorescence staining of splenic lymphocytes in vitro. The double-immunofluorescence staining was performed using anti-CD3, CD79a and EAD-specific monoclonal antibodies. Coexpression of EAD was observed only in some CD79a-positive lymphocytes (a). No lymphocytes coexpressing $\mathrm{EAD}$ and $\mathrm{CD} 3$ were found (b). No EAD expression was observed in EBV-unexposed rabbit lymphocytes (c).

mRNA was detected on days 8 and 10 again. The immunofluorescence staining of splenic lymphocytes on day 4 after the exposure showed EAD-expressing cells (fig. 1d). Ten EAD-positive cells were found in the 468 cells observed, and the abundance of EAD-positive cells was about $2.1 \%$.

Double-Immunofluorescence Staining of EBV-Exposed Splenic Lymphocytes in vitro

We performed double-immunofluorescence staining analysis using anti-CD3 or CD79a antibodies, which were rabbit $\mathrm{T}$ cell and $\mathrm{B}$ cell markers, respectively, and anti-EAD-specific monoclonal antibody (fig. 2). Although CD3- or CD79a-positive lymphocytes were observed by each single staining, some CD79a-positive lymphocytes showed coexpression of EAD (fig. 2a), but the CD3-positive lymphocytes did not (fig. 2b). No EAD-ex-

In vitro EBV Infection of Rabbit Lymphocytes
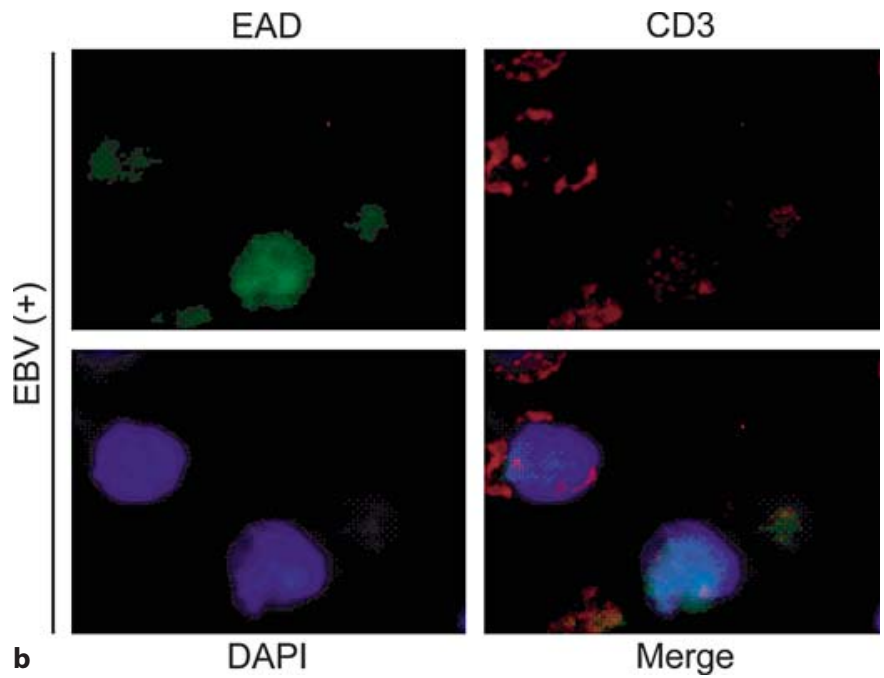

b
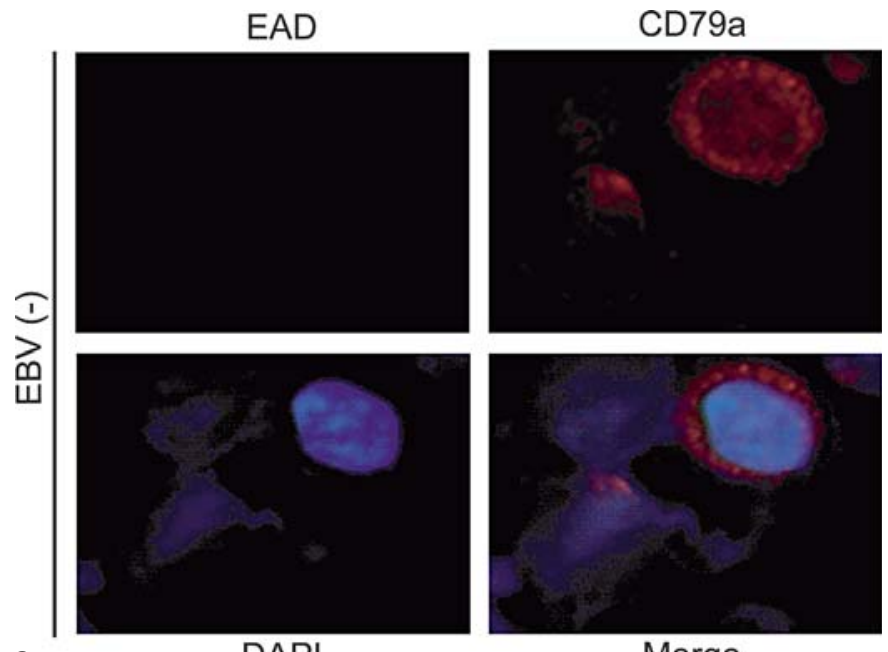

Merge

pressing cells were observed in EBV-unexposed rabbit splenic lymphocytes (fig. 2c).

\section{Colony Formation Assay of EBV-Exposed PBMC in vitro}

Colony formation developed in the dishes plated with EBV-exposed human PBMC on day 11 (data not shown). Colonies also began to appear in dishes plated with EBVinoculated rabbit PBMC 2 weeks later (fig. 3a, b). No colonies developed in dishes plated with EBV-unexposed rabbit PBMC during the 4 weeks of observation.

\section{Flow Cytometric Evaluation of Lymphocyte Subset}

Dynamics Induced by EBV Infection in vivo

Dynamics of lymphocyte subsets in PBMC derived from an EBV-inoculated rabbit were determined by flow cytometry and indicated their increasing rate as relative 
Fig. 3. Colonies obtained from EBV-exposed rabbit PBMC in vitro. Colony formation assay was performed in methylcellulose medium to examine the immortalization potential of EBV-exposed rabbit PBMC. Colonies obtained from plated EBV-exposed rabbit PBMC in the dishes are shown $(\mathbf{a}, \mathbf{b})$. These figures indicate colonies obtained from individual rabbits.
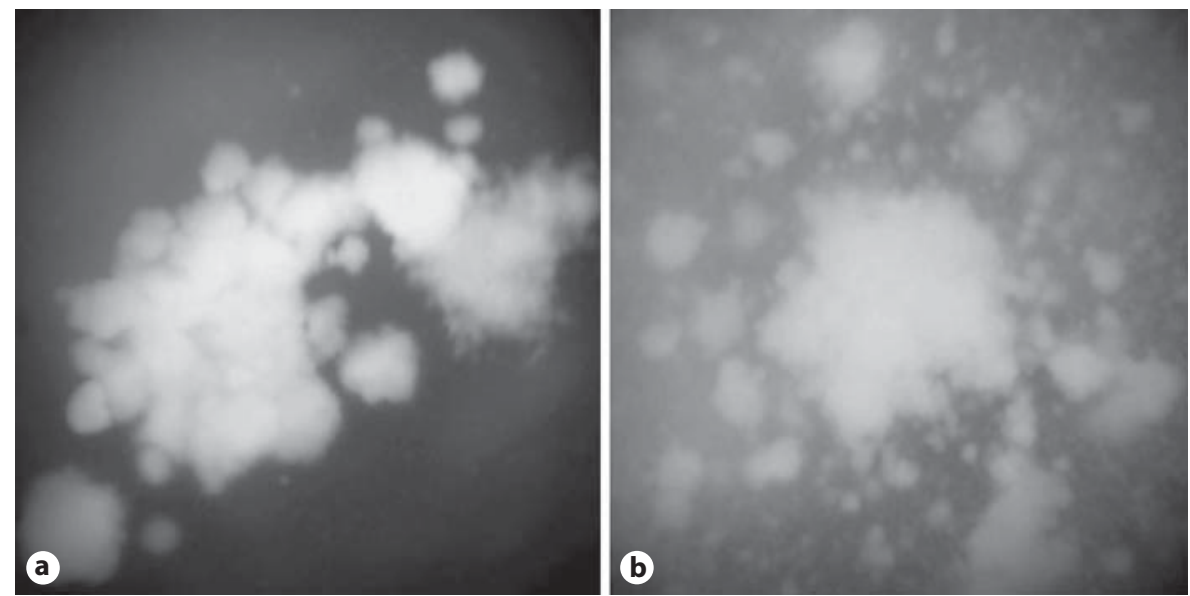

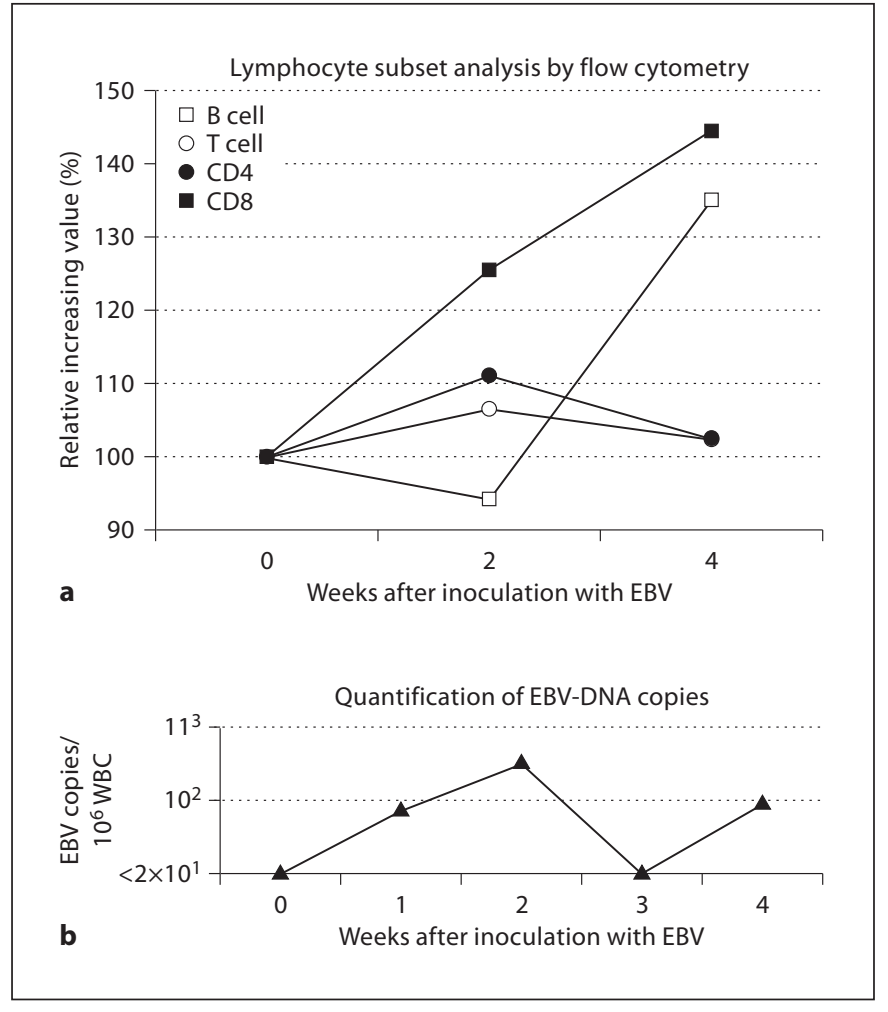

Fig. 4. Transition of each lymphocyte subset and EBV-DNA copy in peripheral blood from EBV-infected rabbits in vivo. Flow cytometry and real-time PCR for PBMC derived from EBV-inoculated rabbits were performed ( $\mathbf{a}$ and $\mathbf{b}$, respectively). Total $\mathrm{T}$ cell and $\mathrm{CD} 4+\mathrm{T}$ cell values were invariable. CD8+ T cells had increased continuously up to $145 \%$ during the observation period. Although B cells decreased to about $95 \%$ by 2 weeks, it increased up to about $135 \%$ after 4 weeks (a). EBV-DNA began to be detected on day 7 after the inoculation, increased by day 14 . Although it disappeared on day 21, EBV-DNA reappeared on day 27 (b). values (fig. 4a). The rabbit was inoculated with EBV intravenously and continuous EBV infection was determined by real-time PCR of EBV-DNA in the peripheral blood (fig. 4b). In this rabbit's case, EBV-DNA began to be detected on day 7 after the inoculation, and increased by day 14 . Although it disappeared on day 21, EBV-DNA reappeared on day 27 . In this period, total $\mathrm{T}$ cells and $\mathrm{CD} 4+\mathrm{T}$ cells were seen as invariable. CD8+ T cells had increased continuously up to $145 \%$ during the observation period. Although the B cell count decreased to about $95 \%$ by 2 weeks, it increased up to about $135 \%$ after 4 weeks.

\section{Discussion}

In vitro rabbit $\mathrm{EBV}$ infection in lymphocytes was confirmed by EBV genes expression in this study. EBV gene transcripts, EBNA1- and ZEBRA-mRNA were observed in EBV-exposed rabbit PBMC on day 2, and EBNA1-, EBNA2- and ZEBRA-mRNA were also detected in the splenic lymphocytes on day 2. However, EBNA2-mRNA were not observed in $\mathrm{PBMC}$, and these negative results may be caused by the lower-positive rate of EBV infection in PBMC. ZEBRA-mRNA reappeared on day 8 in the splenic lymphocytes, but not in PBMC. On the other hand, our previous report showed that one of the EBVinfected rabbits continuously demonstrated ZEBRAmRNA expression over 200 days in the peripheral blood [23]. The reappearance of ZEBRA-mRNA in splenic lymphocytes in vitro may correspond a the similar phenomenon in vivo [23]. These results suggest the possibility of continuous EBV replication in some EBV-infected rabbits. EAD expression was observed in both PBMC and 
splenic lymphocytes, and the abundance of EAD-positive cells was more frequent in splenic lymphocytes than in PBMC. It seemed to be caused by the difference of the abundance of lymphocytes which construct majority of spleens, while lymphocytes are a minor component in PBMC. Similarly, no reappearances of ZEBRA-mRNA in PBMC may be represented by the lower frequency of EBV-infected cells than splenic lymphocytes. This fact also supports the idea that the target cells of rabbit EBV infection were likely to involve lymphocytes. The results of double-immunofluorescence staining to reveal EBVinfected lymphocyte subsets indicated that, at least, EBV can infect some B cells in vitro, because colocalized expression of EAD and CD79a was observed, but not coexpression of EAD and CD3. This result is consistent with in vitro EBV infection in humans in which the main target lymphocyte subset is the B cell, whereas immunohistochemical analyses in our previous report showed EBV infection mainly in B cells and occasionally in T cells in vivo [22]. This difference may be caused by a disparity in the surrounding conditions of EBV-infected cells between in vitro and in vivo conditions as well as the individual differences of the rabbits. However, only rabbit CD3 and CD79a were used as cell markers in this experiment and the EBV infection of other cells is still unknown showing that more investigations are needed. Colony formation assay to reveal immortalized activity of EBV-exposed PBMC derived from two individual rabbits showed colonies of immortalized cells cultured in dishes of EBV-exposed rabbit PBMC. This result suggests that cell immortalization could be induced in EBV-exposed rabbit PBMC. We are trying a culture to establish the lymphoblastoid cell lines that evolve from the colonies of EBV-exposed rabbit PBMC. We are sure that PBMC would be established as lymphoblastic cells, because cell growths have been observed continuously after colony formation assay. We intend to reveal subsets of cells and determine the existence of EBV in cells when a lymphoblastoid cell line would be established. Our previous report on the lifetime observation of EBV-inoculated rabbits demonstrated continuous or intermittent EBVDNA detection in the peripheral blood, and showed lifelong EBV infection conserved in some rabbits without loss of viral functions, because a lot of EBER1-expressing cells were observed in the spleens and lymph nodes at autopsy [unpubl. data]. These facts suggest that EBV in rabbits may also survive through reactivation or immortalization of infected cells similar to humans. However, EBV existence and subset characteristics of colony-forming immortalized cells remain to be elucidated in the future.
To investigate details of infection-related cell dynamics in PBMC of EBV-inoculated rabbits in vivo, although only 1 rabbit has been examined until now, we were successful in establishing a new analyzing system of lymphocyte subsets in EBV-inoculated rabbits by flow cytometry and lymphocyte subset analyses were performed. Total T cells and CD4+ T cells were invariable, but CD8+ T cells increased during the observation period. The increase in CD8+ T cells was likely induced by a reaction of the host immune system against EBV-infected B cells. B cells decreased by 2 weeks and increased up to about $135 \%$ after 4 weeks. EBV-DNA were detected continuously except at 3 weeks. In these experiments, it was shown that reasonable data were obtained from an established analyzing system at this time. This new noninvasive method makes long-term analyses possible, and opens the way for the real-time analyses of rabbit lymphocyte reactions against EBV in combination with quantification of EBV-DNA in the peripheral blood by real-time PCR. Since we revealed that some EBV-inoculated rabbits showed lifetime EBV infection as indicated by detection of EBV-DNA in the peripheral blood through life and because autopsy revealed EBER1 or LMP1 expression in the lymphocytes of the spleen or lymph nodes [unpubl. data], this technique will be beneficial to analyze the mechanisms which cause lifelong EBV infection in rabbits. Though large-scale rabbit experiments are needed to elucidate the exact dynamics of lymphocyte subsets related to rabbit EBV infection in vivo, data obtained from one inoculated rabbit suggest the possibility that $\mathrm{CD} 8+\mathrm{T}$ cells increase to recognize and kill EBV-infected B cells which are the target of EBV infection, and some evaded B cells with EBV infection survive and begin to proliferate after removal of most of the infected cells. The decrease in number of EBV-infected B cells at 2 weeks may be caused by cytotoxic activity of the increased CD8+ T cells, resulting in an undetected level of EBV-DNA at 3 weeks. However, the growth potential of EBV-infected B cells might be higher than the cytotoxic activity of CD8+ T cells, and EBV-infected B cells that evade host immunity could grow at 4 weeks more rapidly than before infection. This might be continued in vivo in this rabbit, because EBV-DNA in the peripheral blood was detected continuously up to 12 weeks (data not shown), as seen by our previous reports that some inoculated rabbits demonstrated continuous EBV infection [23; unpubl. data]. However, there is no direct evidence of the immortalization of rabbit EBV-infected B cells in vivo, and further investigations are needed.

In conclusion, we demonstrated that: (1) EBV can also infect rabbit PBMC and splenic lymphocytes in vitro, be- 
cause EBV gene mRNA expressions in two individual materials from 2 rabbits were confirmed on day 2, and EAD expressions were shown 4 days after EBV exposure. (2) It is highly likely that B cells were the main target cell of rabbit EBV infection and that EBV-exposed cells were immortalized and proliferated similar to humans. (3) CD8+ T cells increased in the rabbit in vivo model after EBV inoculation, whereas an increase of B cells occurred after their transient decrease. These findings suggest that
EBV infection and the subsequent host reactions were induced in the EBV-inoculated rabbits, reconfirming that rabbits are a suitable animal model for EBV infection. Therefore, we demonstrated the experimental systems for the investigation of rabbit EBV infections in vitro. These achievements and in vitro and in vivo rabbit EBV infection systems will be helpful to reveal the mechanisms of EBV infection and the pathogenesis of EBV-associated diseases in the future.

\section{References}

$\checkmark 1$ Epstein MA, Achong BG, Barr YM: Virus particles in cultured lymphoblasts from Burkitt's lymphoma. Lancet 1964;i:702-703.

$\checkmark 2$ Cohen JI: Epstein-Barr virus infection. N Engl J Med 2000;343:481-492.

3 Kimura H, Ito Y, Suzuki R, Nishiyama Y: Measuring Epstein-Barr virus (EBV) load: the significance and application for each EBV-associated disease. Rev Med Virol 2008;18:305-319.

4 Sugimoto M, Tahara H, Ide T, Furuichi Y: Steps involved in immortalization and tumorigenesis in human B-lymphoblastoid cell lines transformed by Epstein-Barr virus. Cancer Res 2004;64:3361-3364.

5 Straus SE, Cohen JI, Tosato G, Meier J: NIH conference. Epstein-Barr virus infections: biology, pathogenesis, and management. Ann Intern Med 1993;118:45-58.

$\checkmark 6$ Imashuku S: Clinical features and treatment strategies of Epstein-Barr virus-associated hemophagocytic lymphohistiocytosis. Crit Rev Oncol Hematol 2002;44:259-272.

7 Straus SE: The chronic mononucleosis syndrome. J Infect Dis 1988;157:405-412.

8 Aozasa K: Pyothorax-associated lymphoma. Int J Hematol 1996;65:9-16.

$\checkmark 9$ Williams H, Crawford DH: Epstein-Barr virus: the impact of scientific advances on clinical practice. Blood 2006;107:862-869.

10 Isaacson P, Wright DH: Malignant lymphoma of mucosa-associated lymphoid tissue: a distinctive type of B-cell lymphoma. Cancer 1983;52:1410-1416.

-11 Johannessen I, Crawford DH: In vivo models for Epstein-Barr virus (EBV)-associated B cell lymphoproliferative disease (BLPD). Rev Med Virol 1999;9:263-277.

-12 Shope T, Dechairo D, Miller G: Malignant lymphoma in cottontop marmosets after inoculation with Epstein-Barr virus. Proc Natl Acad Sci USA 1973;70:2487-2491.

-13 Epstein MA, zur Hausen H, Ball G, Rabin H: Pilot experiments with EB virus in owl monkeys (Aotus trivirgatus). III. Serological and biochemical findings in an animal with reticuloproliferative disease. Int J Cancer 1975; 15:17-22.
14 Rivailler P, Carville A, Kaur A, Rao P, Quink C, Kutok JL, Westmoreland S, Klumpp S, Simon M, Aster JC, Wang F: Experimental rhesus lymphocryptovirus infection in immunosuppressed macaques: an animal model for Epstein-Barr virus pathogenesis in the immunosuppressed host. Blood 2004;104: 1482-1489.

15 Mosier DE, Gulizia RJ, Baird SM, Wilson DB: Transfer of a functional human immune system to mice with severe combined immunodeficiency. Nature 1988;335:256-259.

$\checkmark 16$ Melkus MW, Estes JD, Padgett-Thomas A, Gatlin J, Denton PW, Othieno FA, Wege AK, Haase AT, Garcia JV: Humanized mice mount specific adaptive and innate immune responses to EBV and TSST-1. Nat Med 2006; 12:1316-1322.

17 Yajima M, Imadome K, Nakagawa A, Watanabe $\mathrm{S}$, Terashima $\mathrm{K}$, Nakamura $\mathrm{H}$, Ito $\mathrm{M}$, Shimizu N, Honda M, Yamamoto N, Fujiwara S: A new humanized mouse model of Epstein-Barr virus infection that reproduces persistent infection, lymphoproliferative disorder, and cell-mediated and humoral immune responses. J Infect Dis 2008;198: 673-682.

18 Cocco M, Bellan C, Tussiwand R, Corti D, Traggiai E, Lazzi S, Mannucci S, Bronz L, Palummo N, Ginanneschi C, Tosi P, Lanzavecchia A, Manz MG, Leoncini L: CD34+ cord blood cell-transplanted Rag2 ${ }^{-/-}$gam$\mathrm{ma}(\mathrm{c})^{-1-}$ mice as a model for Epstein-Barr virus infection. Am J Pathol 2008;173:13691378.

19 Hayashi K, Ohara N, Teramoto N, Onoda S, Chen HL, Oka T, Kondo E, Yoshino T, Takahashi K, Yates J, Akagi T: An animal model for human EBV-associated hemophagocytic syndrome: herpesvirus papio frequently induces fatal lymphoproliferative disorders with hemophagocytic syndrome in rabbits. Am J Pathol 2001;158:1533-1542.
20 Hayashi K, Jin Z, Onoda S, Joko H, Teramoto N, Ohara N, Oda W, Tanaka T, Liu YX, Koirala TR, Oka T, Kondo E, Yoshino T, Takahashi K, Akagi T: Rabbit model for human EBV-associated hemophagocytic syndrome (HPS): sequential autopsy analysis and characterization of IL-2-dependent cell lines established from herpesvirus papio-induced fatal rabbit lymphoproliferative diseases with HPS. Am J Pathol 2003;162:17211736 .

21 Hayashi K, Joko H, Koirala TR, Onoda S, Jin ZS, Munemasa M, Ohara N, Oda W, Tanaka T, Oka T, Kondo E, Yoshino T, Takahashi K, Yamada M, Akagi T: Therapeutic trials for a rabbit model of EBV-associated hemophagocytic syndrome (HPS): effects of vidarabine or CHOP, and development of herpesvirus papio (HVP)-negative lymphomas surrounded by HVP-infected lymphoproliferative disease. Histol Histopathol 2003;18: 1155-1168.

22 Takashima K, Ohashi M, Kitamura Y, Ando K, Nagashima K, Sugihara H, Okuno K, Sairenji T, Hayashi K: A new animal model for primary and persistent Epstein-Barr virus infection: human EBV-infected rabbit characteristics determined using sequential imaging and pathological analysis. J Med Virol 2008;80:455-466

23 Okuno K, Takashima K, Kanai K, Ohashi M, Hyuga H, Sugihara H, Kuwamoto S, Kato M, Sano H, Sairenji T, Kanzaki S, Hayashi K: Epstein-Barr virus can infect rabbits by perioral or intranasal route: an animal model for natural primary EBV infection in humans. J Med Virol, in press.

24 Baer R, Bankier AT, Biggin MD, Deininger PL, Farrell PJ, Gibson TJ, Hatfull G, Hudson GS, Satchwell SC, Seguin C, Tuffnell PS, Barrell BG: DNA sequence and expression of the B95-8 Epstein-Barr virus genome. Nature 1984;310:207-211.

25 Kimura H, Morita M, Yabuta Y, Kuzushima K, Kato K, Kojima S, Matsuyama T, Morishima T: Quantitative analysis of Epstein-Barr virus load by using a real-time PCR assay. J Clin Microbiol 1999;37:132-136. 